\title{
Mathematical model of blood glucose dynamics by emulating the pathophysiology of glucose metabolism in type 2 diabetes mellitus
}

\section{CURRENT STATUS: POSTED}

Research Square

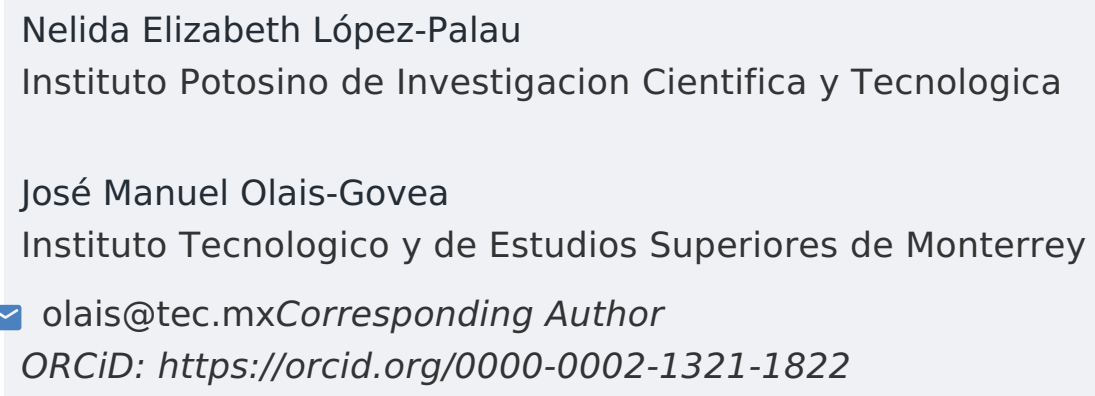

DOI:

10.21203/rs.3.rs-17229/v1

\section{SUBJECT AREAS}

Applied Mathematics

\section{KEYWORDS}

type 2 diabetes pathophysiology, compartmental mathematical models, pharmacokinetic-pharmacodynamic modeling approach, parameter fitting 
Abstract

This work addresses a physiological-based mathematical model of blood glucose dynamics in Type 2 Diabetes Mellitus (T2DM). The model emulates the pathophysiology of the T2DM metabolism including the gastric emptying effect and the enhancing effect on insulin due to the incretin hormones. Its mathematical structure considers a model of blood glucose dynamics of healthy humans developed with a physiological-based pharmacokinetic-pharmacodynamic approach. Then, the mathematical functions, representing the metabolic rates with a relevant contribution to hyperglycemia, are individually fitted to clinical data of T2DM patients. Methodologically, it allows emulating the pathophysiology of the T2DM condition. Numerically, the resulting model simulates successfully a programmed graduated intravenous glucose test and different-doses oral glucose tolerance tests. The comparison between simulations and clinical data shows a good agreement description of the blood glucose dynamics in T2DM and, it opens the likelihood of using this model to develop model-based controllers for blood glucose regulation in T2DM.

Full Text

Due to technical limitations, full-text HTML conversion of this manuscript could not be completed. However, the manuscript can be downloaded and accessed as a PDF.

\section{Tables}

Due to technical limitations, the tables are only available as a download in the supplemental files section.

Figures 

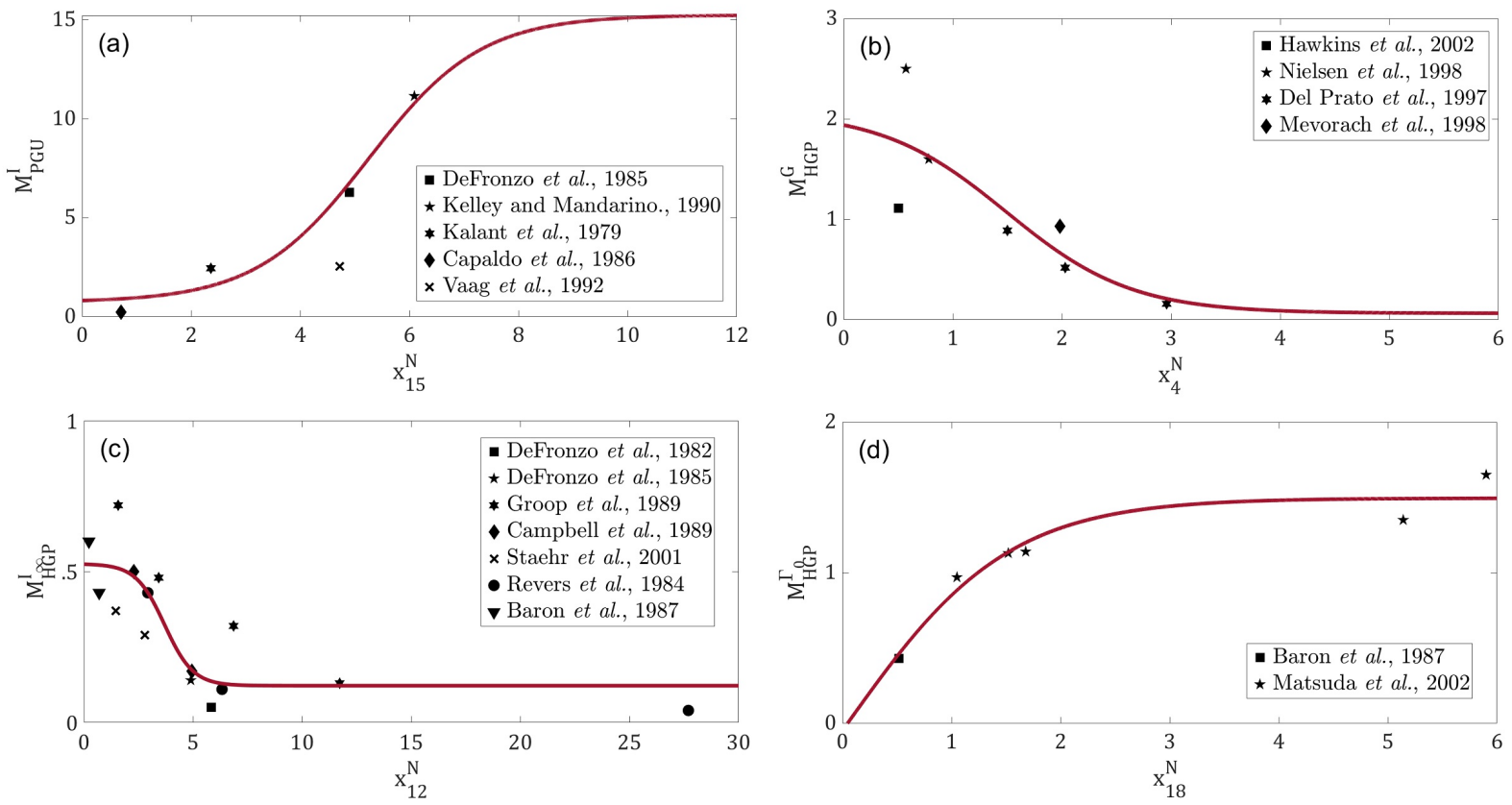

Figure 1

Graphical representation of the isolated effects fitted to clinical data of T2DM patients. The solid line represents the isolated efects of (a) MIPGU , (b) MGHGP, (c) MI¥ HGP and (d) MG0 HGP after parameter fitting. 

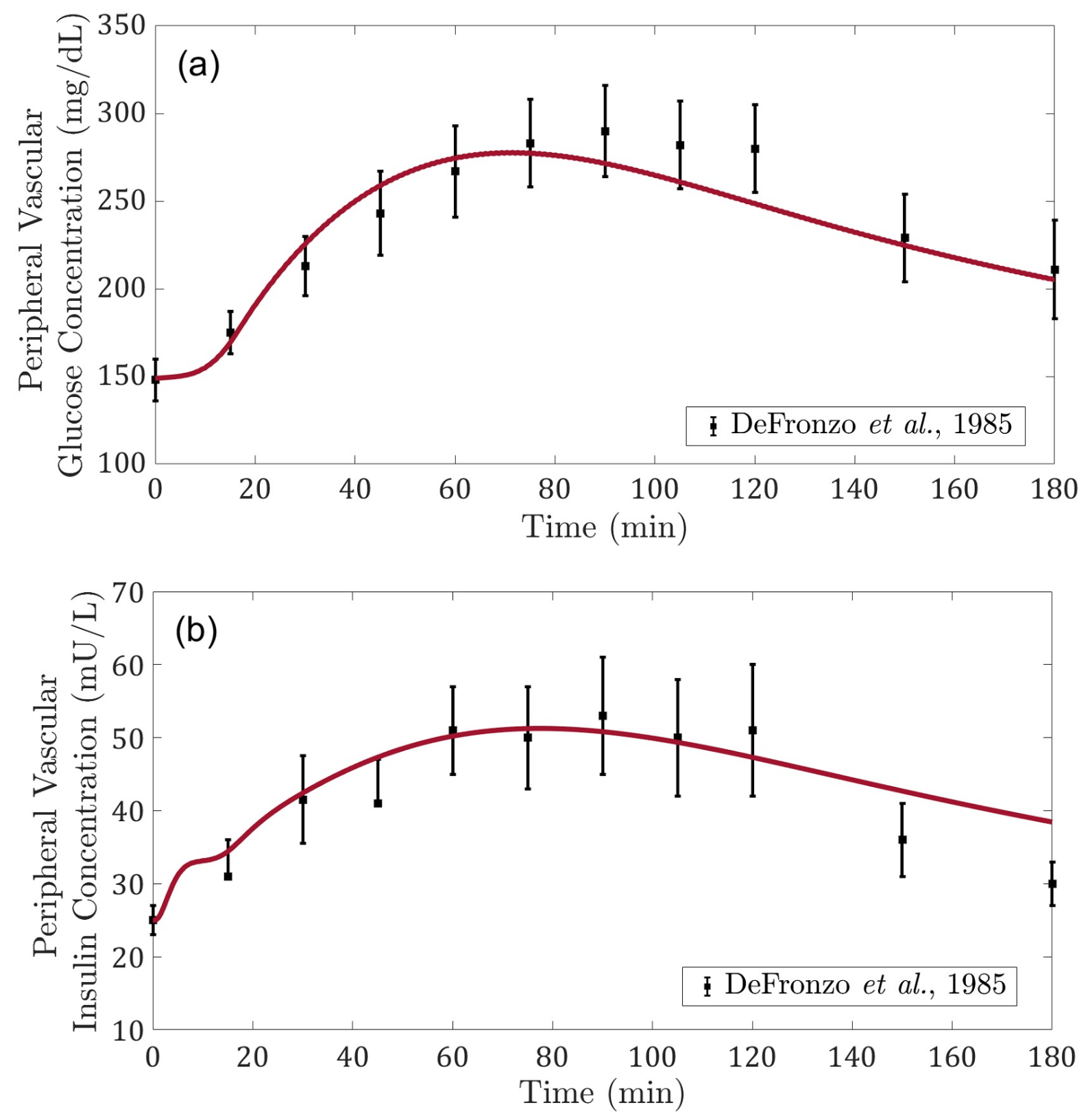

Figure 2

Graphical representation of the $70 \mathrm{~g}-\mathrm{OGTT}$. The model considering the $\theta^{\wedge}$ parameters simulate the blood glucose dynamics after the ingestion of $70 \mathrm{~g}$ of glucose at time zero. The solid line represents the model response $((a) \times 6$ and $(b) \times 14)$, while the black squares represents the clinical data used to identify the selected parameters of rPIR and taken from DeFronzo et al., [25]. 


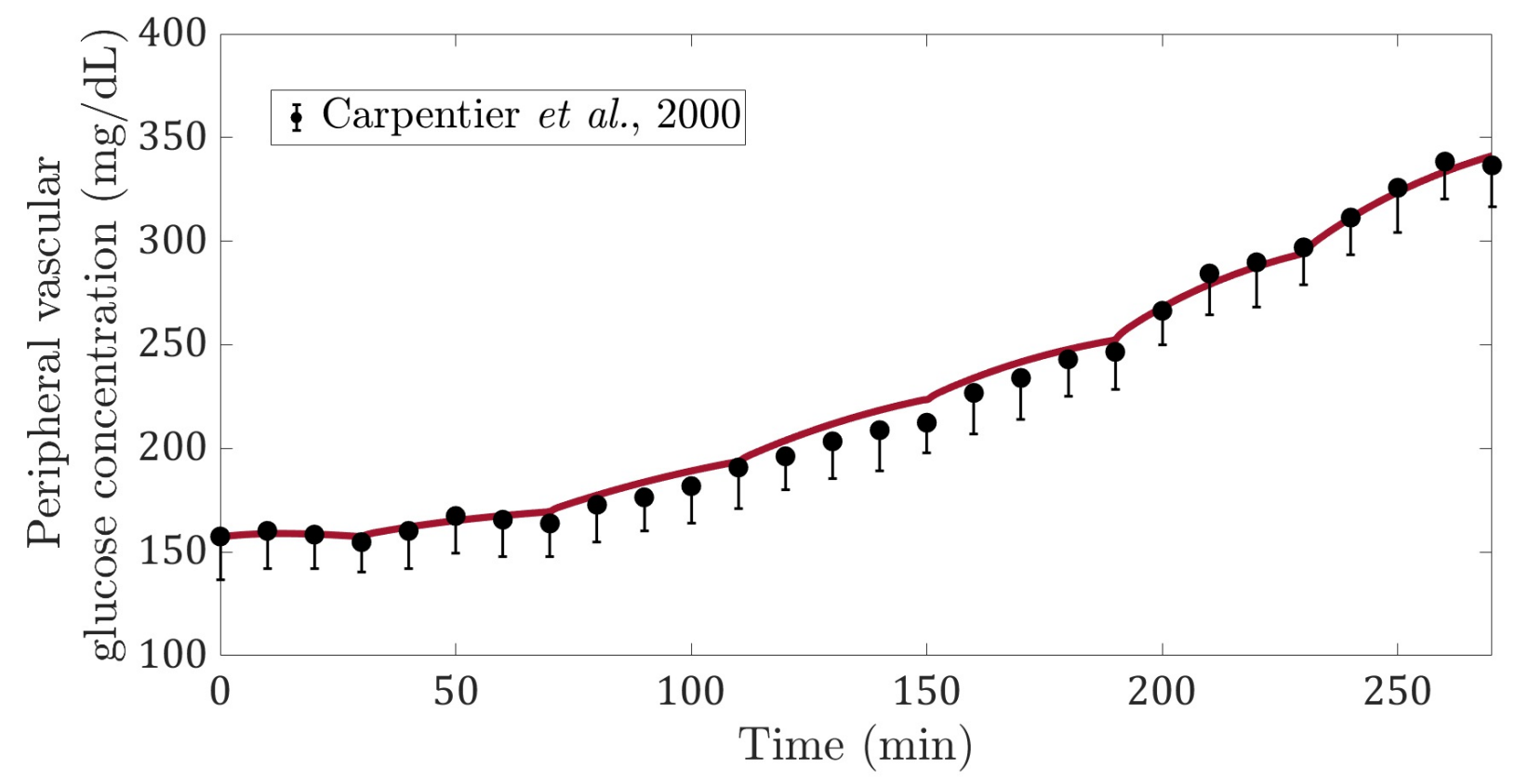

Figure 3

Graphical representation of the PGIGI. The T2DM simulate the blood glucose dynamics after the intravenous infusion of glucose at step graded rates. The solid line represents the T2DM model response $(x 6)$, while the black squares represents the clinical data from Carpentier et al. [27]. 


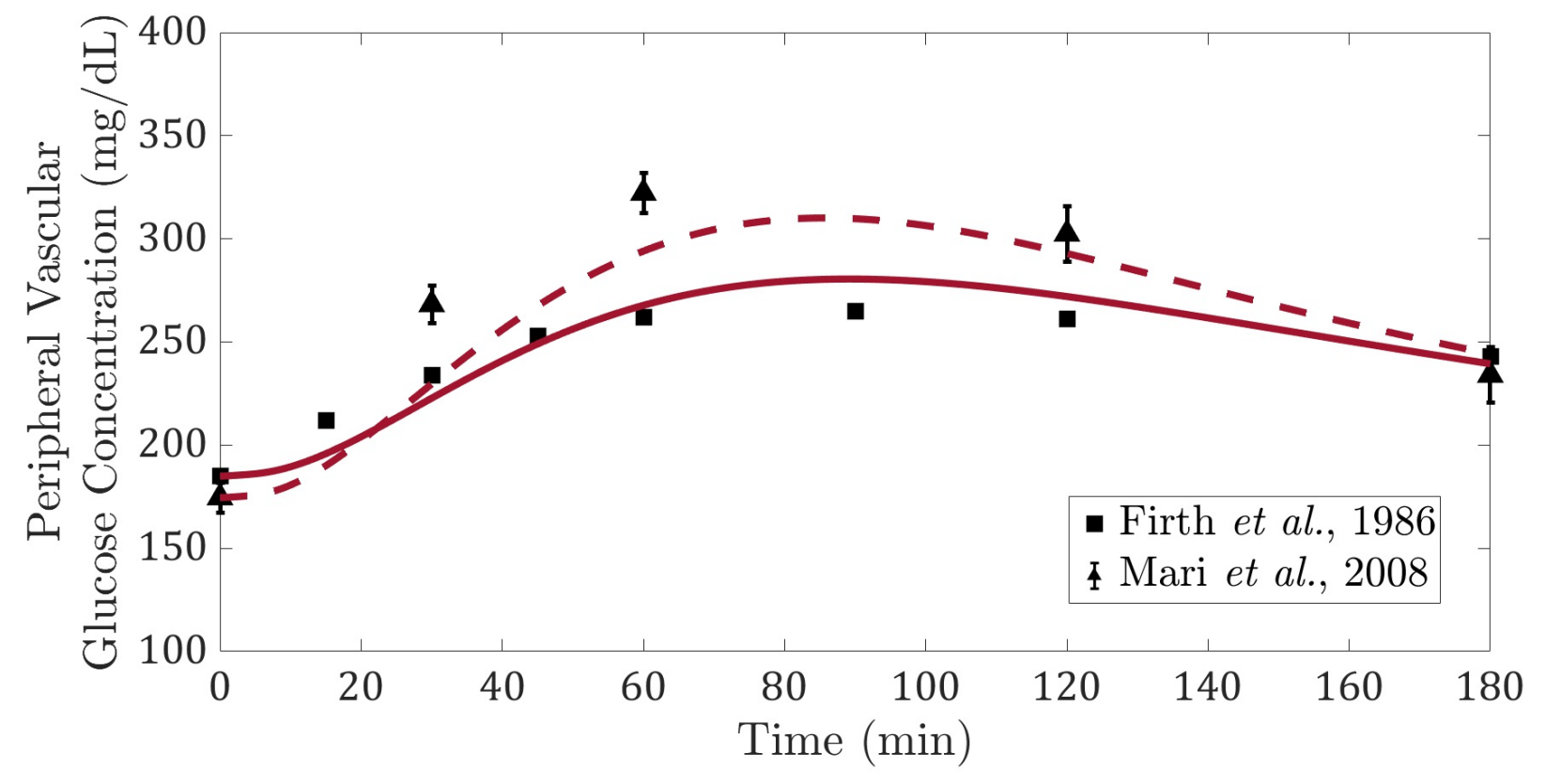

Figure 4

Graphical representation of a 25g-OGTT and a 75g-OGTT. The T2DM model simulated the blood glucose dynamics after the ingestion of the respective glucose dose at time zero. The solid and dashed lines represents the model response (x6) for the $25 \mathrm{~g}$-OGTT and a $75 \mathrm{~g}$ OGTT, respectivelly, while the black triangles and squares represents the clinical data from Mari et al. [29] and Firth et al. [28], respectively. 


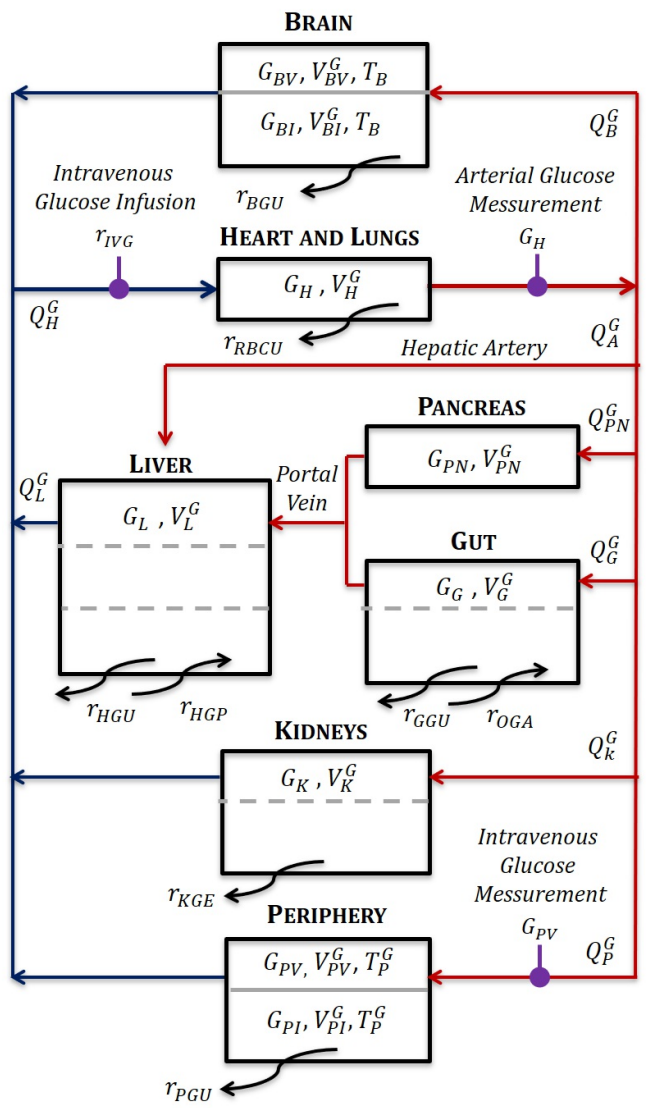

(a)

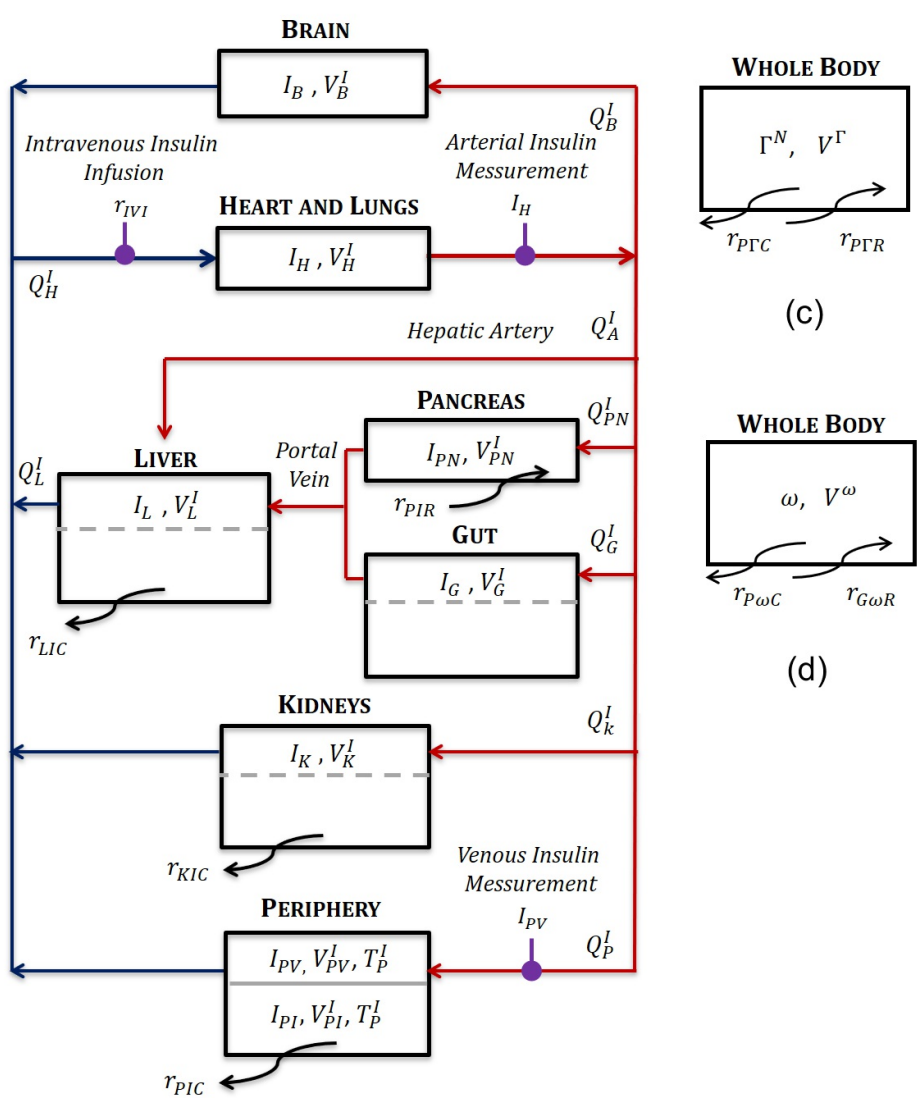

(b)

Figure 5

Model Subsystems.- The compartmental diagrams of the (a) glucose, (b) insulin, (c)

glucagon and (d) incretins subsystems are shown. The continuous line dividing the subcompartments is used to indicate a low permeability between the walls (i.e. variable mass concentration). On the other hand, the dashed line indicate a high permeability, therefore all the sub-compartments in these compartments can be considered as one (i.e. uniform mass concentration). 


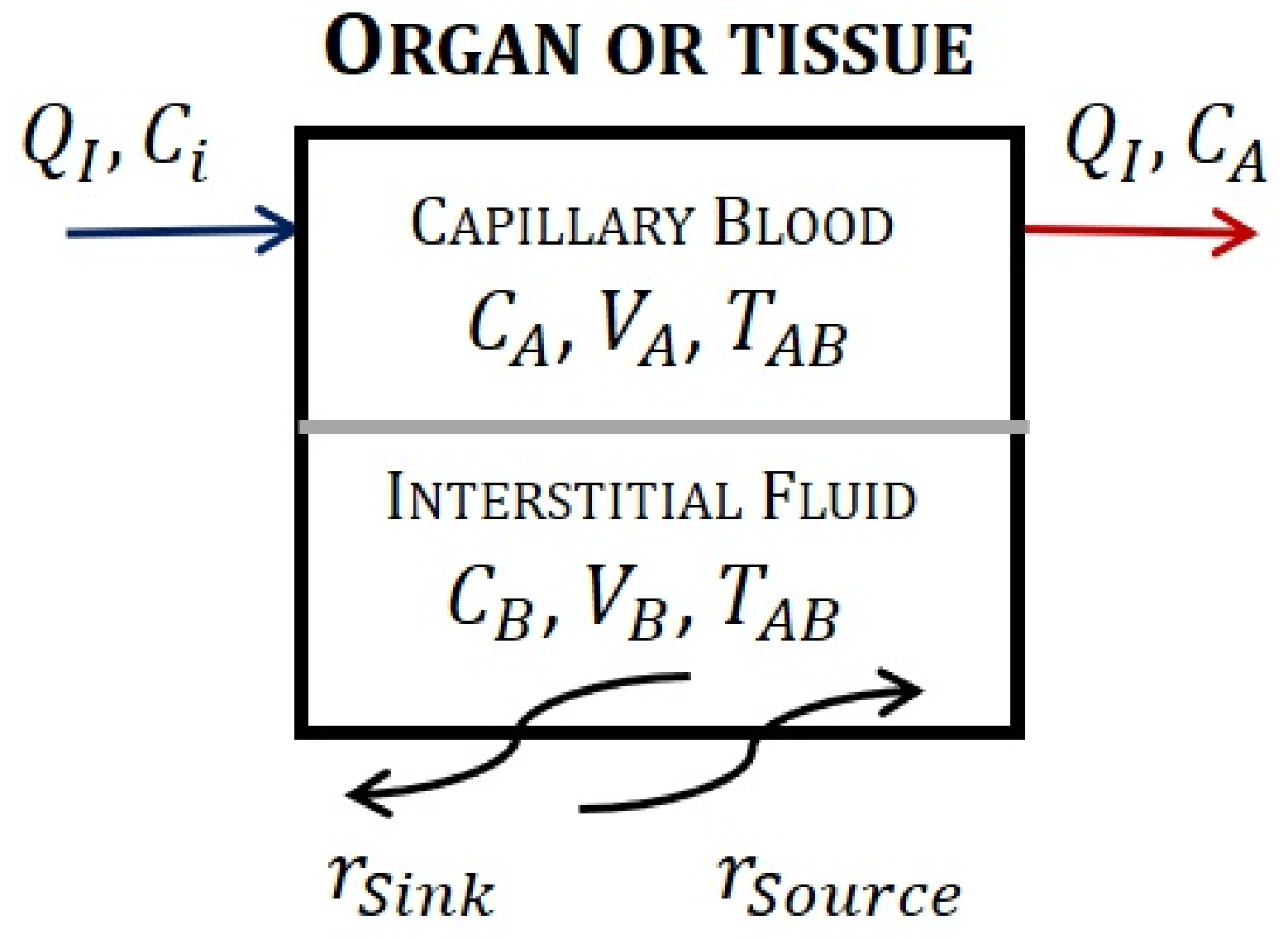

Figure 6

General scheme of a compartment. In this representation, there are two well-dened spaces:

the capillary blood space and the interstitial uid space. Among them, there is a low permeability determined by the transcapillary diffusion time TAB. The metabolic rates that add or eliminate the mass of the subsystems are represented by curved arrows entering or leaving the compartments, respectively.

Supplementary Files

This is a list of supplementary files associated with this preprint. Click to download.

Table 8f.xls

Table 5f.xls

Table 2f.xls

Table 1f.xls

Table 6f.xls

Table 4f.xls

Table 7f.xls

Table 3f.xls 
